\title{
Article
}

Mycosphere

\section{Does morphology matter in taxonomy of Lasiodiplodia? An answer from Lasiodiplodia hyalina sp. nov.}

\author{
Dou $\mathrm{ZP}^{1}$, He $\mathrm{W}^{2}$, Zhang $\mathrm{Y}^{1}$ \\ ${ }^{1}$ Institute of Microbiology, PO Box 61, Beijing Forestry University, Beijing 100083, PR China \\ ${ }^{2}$ Beijing Key Laboratory for Forest Pest Control, Beijing Forestry University, Beijing 100083, PR China
}

Dou ZP, He W, Zhang Y 2017 - Does morphology matter in taxonomy of Lasiodiplodia? An answer from Lasiodiplodia hyalina sp. nov. Mycosphere 8(8), 1014-1027, Doi $10.5943 /$ mycosphere/8/2/5

\begin{abstract}
A new species of Lasiodiplodia (L. hyalina) is described and illustrated from Acacia confusa and an unidentified woody plant collected in Southern China. Only asexual states of $L$. hyalina were observed, which is characterized by most conidia remaining hyaline with only about $10 \%$ conidia becoming pigmented after three months in culture. Phylogenetically, L. hyalina is closely related to $L$. thailandica. Morphologically, the larger conidiogenous cells and paraphyses of L. hyalina are distinct from those of L. thailandica, which leads to the conclusion that the collected taxon is new to science. Lasiodiplodia thailandica is reported as a new record in China with Podocarpus macrophyllus and Albizia chinensis as its new hosts.
\end{abstract}

Key words - Botryosphaeriaceae - China - hyaline conidia - phylogeny

\section{Introduction}

Lasiodiplodia Ellis \& Everh. was formally introduced in Clendenin (1896), and was typified by L. theobromae (Pat.) Griffon \& Maubl. (Phillips et al. 2013). Lasiodiplodia had been considered as a possible synonym of Diplodia Fr. (Denman et al. 2000), while the presence of pycnidial paraphyses, longitudinal striations on mature conidia, and the results of phylogenetic studies suggest that it separates from Diplodia as a well-defined genus (Sutton 1980, Zhou \& Stanosz 2001, Slippers et al. 2004, Phillips et al. 2008, 2013, Prasher \& Singh 2014). Although the morphological characteristics of Lasiodiplodia spp. are quite comparable, features of pycnidia, conidia and paraphyses have been widely used in distinguishing Lasiodiplodia from other genera of Botryosphaeriaceae as well as distinguishing different species within Lasiodiplodia (Phillips et al. 2013, Slippers et al. 2017).

So far, five Lasiodiplodia species have been reported in China, namely L. chinensis Z. P. Dou, Y. Zhang ter., L. hormozganensis Abdollahz., Zare \& A.J.L. Phillips, L. iraniensis Abdollahz., Zare \& A.J.L. Phillips, L. pseudotheobromae A.J.L. Phillips, A. Alves \& Crous and L. theobromae (Zhao et al. 2010, Luo et al. 2011, Li et al. 2015, Dou et al. 2017). During an opportunistic collection of ascomycetous fungi in Southern China, one new taxon with general characteristics of Lasiodiplodia was collected. Combined ITS, tefl- $\alpha$, TUB and RPB2 DNA sequence comparisons verified its new status within Lasiodiplodia. Based on the combination of morphological and molecular differences, a new species, L. hyalina, is introduced. Lasiodiplodia 
thailandica is collected from Podocarpus macrophyllus D. Don and Albizia chinensis Merr., which is reported as a new record in China herein.

\section{Materials \& Methods}

\section{Isolates and morphology}

Cankered branches of Acacia spp., Podocarpus spp., Albizia spp. as well as some unidentified tree species were collected in Guangdong and Hainan Province, China during November 2015 and January 2016. Wood segments of $0.5 \mathrm{~cm} \times 0.5 \mathrm{~cm} \times 0.2 \mathrm{~cm}$ were cut from the canker lesion boundary. The wood segments were then surface sterilized (Pavlic et al. 2004) and cultured on malt extract agar (MEA) for fungal strains. Plates were incubated at $28{ }^{\circ} \mathrm{C}$ under continuous near-UV light for two weeks and colonies resembling Lasiodiplodia spp. were selected and transferred to synthetic nutrient-poor agar (SNA). Isolates were maintained on $2 \%$ MEA at 28 ${ }^{\circ} \mathrm{C}$ and stored at $4{ }^{\circ} \mathrm{C}$. Isolates grown on MEA were kept at ambient temperatures (about $28{ }^{\circ} \mathrm{C}$ ) in the dark to establish colony characteristics. Fungal isolates were deposited at Beijing Forestry University (BJFU) with duplicates in the China General Microbiological Culture Collection Center (CGMCC) and the Mycological Herbarium of the Institute of Microbiology, Chinese Academy of Sciences (HMAS).

To induce sporulation, isolates were grown on $2 \%$ water agar (WA) (Biolab, S.A.) with sterilized pine needles placed onto the medium, at $28{ }^{\circ} \mathrm{C}$ under near-UV light. Released conidia and squash mounts of pycnidia formed on the pine needles, were mounted in water on microscope slides and examined microscopically. Measurements and digital photographs were made using a Nikon Coolpix 995 digital camera connected to a trinocular Leitz Orthoplan microscope and processed with Adobe Photoshop Elements 10 software. Measurements of conidia, paraphyses and conidiogenous cells were made from water mounts.

\section{DNA extraction, PCR amplification}

DNA was extracted from mycelium grown on MEA plates with CTAB plant genome DNA fast extraction kit (Aidlab Biotechnologies Co., Ltd, Beijing, China). The internal transcribed spacer of rDNA (ITS) was amplified and sequenced with primers ITS-1 and ITS-4 (White et al. 1990). The translation elongation factor- $1 \alpha$ (tefl- $\alpha)$ was amplified and sequenced with primers EF1-688F and EF1-1251R (Alves et al. 2008). The $\beta$-tubulin gene (TUB) was amplified and sequenced with primers Bt2a and Bt2b (Glass \& Donaldson 1995). The RPB2 sequences were amplified and sequenced using primers RPB2-LasF and RPB2-LasR (Cruywagen et al. 2017). PCR amplification and sequencing followed the protocol of Zhang et al. (2009).

\section{Sequence alignment and phylogenetic analysis}

The combined loci of ITS, tefl- $\alpha, T U B$ and RPB2 were used to infer the phylogenetic relationships among different species of Lasiodiplodia by maximum parsimony (MP) and MrBayes analyses. Sequences generated were analyzed with other sequences obtained from GenBank (Table 1). Alignments were conducted in MEGA v. 6 (Tamura et al. 2013) and phylogenetic analyses performed in PAUP v. 4.0b10 (Swofford 2002) and MrBayes v. 3.1.2 (Ronquist \& Huelsenbeck 2003). Prior to phylogenetic analysis, ambiguous sequences at the start and the end were deleted and gaps manually adjusted to optimize the alignments. Maximum Parsimony (MP) was used to conduct heuristic searches as implemented in PAUP with the default options method (Zhang et al. 2008). Analyses were done under different parameters of maximum parsimony criteria as outlined in Zhang et al. (2008). Clade stability was assessed in a bootstrap analysis with 1000 replicates, random sequence additions with maxtrees set to 1000 and other default parameters as implemented in PAUP. For the MrBayes analysis, the best-fit model of nucleotide evolution $(\mathrm{GTR}+\mathrm{I}+\mathrm{G})$ was selected by Akaike information criterion (AIC; Posada \& Buckley 2004) in MrModeltest v. 2.3. The metropolis-coupled Markov Chain Monte Carlo (MCMCMC) approach was used to calculate posterior probabilities (Huelsenbeck \& Ronquist 2005). A preliminary Bayesian inference (BI) 
analysis using MrBayes software revealed that the Markov Chain Monte Carlo (MCMC; Huelsenbeck \& Ronquist 2001) steady state was reached after less than 10,000 generations (the average standard deviation of split frequencies was constantly below 0.01). A conservative burn-in of 100 trees was chosen and a full analysis of 12,000,000 generations was carried out with sampling every 100 generations. Trees were viewed in TREEVIEW. The nucleotide sequences generated in this paper were deposited in GenBank (Table 1). Trees and alignments were deposited in TreeBase (S20654).

\section{Results}

\section{Phylogenetic analyses}

Phylogenetic analysis of the combined ITS, tefl- $\alpha, T U B$ and RPB2 sequence dataset comprising $1957 \mathrm{bp}$ revealed 301 parsimony-informative characters. The outgroup taxon was Diplodia mutila and D. seriata. The heuristic search with random addition of taxa (1,000 replicates) generated 5000 most parsimonious trees of 771 steps $(\mathrm{CI}=0.638, \mathrm{RI}=0.864, \mathrm{RC}=0.551, \mathrm{HI}=$ 0.362). In the phylogenetic tree, the clade comprising L. thailandica, L. hyalina and L. iraniensis received high support for both Bayesian and MP analysis (Fig. 1). The subclades comprising individual species of $L$. thailandica and L. iraniensis both received high Bayesian analysis support, and moderate support in MP analysis.

\section{Taxonomy}

Lasiodiplodia hyalina Z. P. Dou, Y. Zhang, sp. nov.

Mycobank No.: MB 817651; Facesoffungi number: FoF03151.

Etymology - from the Latin "hyaline", in reference to the hyaline conidia.

Sexual morph unknown. Asexual morph: Conidiomata stromatic, produced on pine needles on SNA within 1-2 wk, solitary, immersed or semi-immersed, iron grey to black, covered with dense mycelium, mostly uniloculate, 255-500 $\mu \mathrm{m}$ diam, solitary, globose, thick-walled, with a central ostiole. Paraphyses hyaline, cylindrical, thin-walled, initially aseptate, becoming up to 1-7 septate when mature, sometimes branched or connected to the ladder shaped or $\mathrm{H}$ form, rounded at apex, occasionally basal or apical cells swollen, 24-82 × 3-7 $\mu \mathrm{m}$. Conidiophores absent. Conidiogenous cells holoblastic, discrete, hyaline, smooth, thin-walled, cylindrical to ampulliform, proliferating percurrently, $(8-) 9-18(-20) \times 4-7 \mu \mathrm{m}(\mathrm{av} .=12.6 \times 5.3 \mu \mathrm{m}, \mathrm{n}=60)$. Conidia initially hyaline, aseptate, ellipsoid to ovoid, occasionally with a median or submedian constriction, including granular content, both ends broadly rounded, thick-walled, verruculose, (19-)20-27(-28) × 12-16 $\mu \mathrm{m}$ (av. of 30 conidia $=24 \times 13.6 \mu \mathrm{m}, \mathrm{L} / \mathrm{W}$ ratio $=1.77$, range from 1.36 to 2.00 ), a few conidia turning pale brown with a single median septum and longitudinal striations after three months, but most conidia remain hyaline.

Culture characteristics - Colonies on MEA initially white with woolly aerial mycelia, becoming iron grey to black on the surface after 2 weeks; reverse side of the colonies olivaceousgrey to dark black. Colonies reaching $76.5 \mathrm{~mm}$ on MEA after $48 \mathrm{~h}$ in the dark at $28{ }^{\circ} \mathrm{C}$.

Specimens examined - CHINA, Hainan Province, Danzhou City, the Danzhou Tropical Botanical Garden, from cankered stems of Acacia confusa Merr., 3 November 2015, Y. Zhang \& Y. P. Zhou (HMAS 255216, holotype), ex-type living culture, CGMCC 3.17975; Guangdong Province, Guangzhou City, Sculpture Park, from cankered branches of an unidentified woody plant, 21 January 2016, Z.P. Dou \& Z.C. Liu (CGMCC 3.18383).

Note - The conidia of L. hyalina keep hyaline until three months' growing on SNA, then only a small proportion (ca. 10\%) of conidia become pigmented with striations on the surface, which looks senescent. Phylogenetically, L. hyalina is closely related to L. thailandica and L. iraniensis (Fig.1). The tef1- $\alpha$ region of L. hyalina is distinguishable from those of L. thailandica (CPC 22795, GenBank Accession No. KJ193681) and L. iraniensis (IRAN 1520C, GenBank Accession No. GU945336) with identity of $97.0 \%$ and $97.1 \%$, respectively. In addition, the larger conidiogenous cells $((8-) 9-18(-20) \times 4-7 \mu \mathrm{m} v s 8-9 \times 2-4 \mu \mathrm{m})$ and broader $(3-7 \mu \mathrm{m} v s 1-1.5 \mu \mathrm{m})$, branching and 
anastomosing paraphyses of $L$. hyalina are distinguishable from those of $L$. thailandica (Trakunyingcharoen et al. 2015). The smaller conidiomata $(255-500 \mu \mathrm{m} v s$ up to $980 \mu \mathrm{m})$ and shorter paraphyses $(24-82 \mu \mathrm{m} v s$ up to $127 \mu \mathrm{m})$ of L. hyalina differs from those of L. iraniensis (Abdollahzadeh et al. 2010). Furthermore, some hyaline conidia of L. hyalina show a median or submedian constriction (Fig.2), which also distinguishes it from the other two species.

Lasiodiplodia thailandica T. Trakunyingcharoen, L. Lombard \& Crous, in Trakunyingcharoen, Lombard, Groenewald, Toanun \& Crous, Persoonia 34: 95 (2015)

Specimens examined - CHINA, Guangdong Province, Guangzhou City, Baiyun Mountain, from cankered branch of Podocarpus macrophyllus, 19 January 2016, Z.P. Dou \& Z.C. Liu (CGMCC 3.18382). Yangchun City, Kongtongyan Scenic Area, from cankered branch of Albizia chinensis, 23 January 2016, Z.P. Dou \& Z.C. Liu (CGMCC 3.18384).

\section{Discussion}

Morphological characteristics of sexual or asexual stage have their weakness in taxonomy of Botryosphaeriaceae, while their significance cannot be ignored (Phillips et al. 2013, Slippers et al. 2014, 2017). Morphologically, the striations on the pigmented conidia and the presence of conidiomatal paraphyses distinguish Lasiodiplodia from all other genera of Botryosphaeriaceae, while the morphology of pycnidia or conidia (especially dimensions), as well as morphology of the paraphyses can be used in species identification of Lasiodiplodia spp (Table 2, Phillips et al. 2013). For instance, the large-sized, 1-3-septate mature conidia and aseptate paraphyses of L. gonubiensis Pavlic, Slippers \& M.J. Wingf. could be distinguishable from other reported species of Lasiodiplodia, and L. rubropurpurea T.I. Burgess, Barber \& Pegg could be recognized based on its unique livid red to dark vinaceous pycnidia (Pavlic et al. 2004, Burgess et al. 2006, Alves et al. 2008). Although the morphology of Lasiodiplodia species differ from each other in some degree, the identification of Lasiodiplodia species cannot be safely applied without the help of related DNA sequence comparisons.

Phylogenetically, L. hyalina forms a robust clade with L. thailandica (Fig. 1). Conidia of both L. hyalina and L. thailandica tend to keep hyaline, and only a small proportion of the discharged conidia getting pigmented with age (Trakunyingcharoen et al. 2015), which differs from most other reported species of Lasiodiplodia. Although no pigmented conidia were produced in L. sterculiae Tao Yang \& Crous after being cultured for two months in SNA medium, it lacks of the description about aged conidia for this species (Yang et al. 2017). Thus, the conidia pigmentation of $L$. sterculiae cannot be determined until information about the aged conidia was provided after longer incubation. So far, the one (or rarely up to three) septum, pigmented conidia with striations on its surface (sometimes for aged conidia) can serve as diagnosing characteristics for Lasiodiplodia yet.

Lasiodiplodia thailandica was first described from symptomless twigs of Mangifera indica from Thailand (Trakunyingcharoen et al. 2015), and it was retrieved from the cankered branches of Podocarpus macrophyllus and Albizia chinensis in tropical region of China. It seems that $L$. thailandica is a tropical species with a wide range of host spectrum. Podocarpus spp. seem to be good hosts for fungi, as many new fungal taxa has been reported from the genus (Dai et al. 2009, 2010, 2011, Zhou \& Dai 2013). 
Table 1 GenBank and culture collection accession numbers of species included in the phylogenetic study. Newly deposited sequences are shown in bold.

\begin{tabular}{|c|c|c|c|c|c|c|c|}
\hline \multirow[t]{2}{*}{ Species } & \multirow[t]{2}{*}{ Cultures } & \multirow[t]{2}{*}{ Host } & \multirow[t]{2}{*}{ Locality } & \multicolumn{4}{|l|}{ GenBank } \\
\hline & & & & ITS & tef1-a & $T U B$ & RPB2 \\
\hline Diplodia mutila & CMW 7060 & Fraxinus excelsior & Netherlands & AY236955 & AY236904 & AY236933 & EU339574 \\
\hline D. seriata & CBS 112555 & Vitis vinifera & Portugal & AY259094 & AY573220 & DQ458856 & N/A \\
\hline L. avicenniae & CMW 41467 & Avicennia marina & South Africa & KP860835 & KP860680 & KP860758 & KU587878 \\
\hline L. avicenniae & LAS 199 & Avicennia marina & South Africa & KU587957 & KU587947 & KU587868 & KU587880 \\
\hline L. brasiliense & CMM 4015 & Mangifera indica & Brazil & JX464063 & JX464049 & N/A & N/A \\
\hline L. brasiliense & CMM 2321 & Carica papaya & Brazil & KC484797 & KC481528 & N/A & N/A \\
\hline L. brasiliense & CMW 35884 & Adansonia madagascariensis & Madagascar & KU887094 & KU886972 & KU887466 & KU696345 \\
\hline L. bruguierae & CMW 41470 & Bruguiera gymnorrhiza & South Africa & KP860833 & KP860678 & KP860756 & KU587875 \\
\hline L. bruguierae & CMW 42480 & Bruguiera gymnorrhiza & South Africa & KP860832 & KP860677 & KP860755 & KU587876 \\
\hline L. caatinguensis & CMM 1325 & Citrus sinensis & Brazil & KT154760 & KT008006 & KT154767 & N/A \\
\hline L. caatinguensis & IBL 381 & Spondias purpurea & Brazil & KT154757 & KT154751 & KT154764 & N/A \\
\hline L. chinensis & CGMCC 3.18061 & unknown & China & KX499889 & KX499927 & KX500002 & KX499965 \\
\hline L. chinensis & CGMCC 3.18044 & Vaccinium uliginosum & China & KX499875 & KX499913 & KX499988 & KX499951 \\
\hline L. chinensis & CGMCC 3.18066 & Hevea brasiliensis & China & KX499899 & KX499937 & KX500012 & KX499974 \\
\hline L. chinensis & CGMCC 3.18067 & Sterculia lychnophora & China & KX499901 & KX499939 & KX500014 & KX499976 \\
\hline L. citricola & IRAN 1522C & Citrus sp. & Iran & GU945354 & GU945340 & KU887505 & KU696351 \\
\hline L. citricola & IRAN 1521C & Citrus sp. & Iran & GU945353 & GU945339 & KU887504 & KU696350 \\
\hline L. crassispora & WAC 12533 & Santalum album & Australia & DQ103550 & DQ103557 & KU887506 & KU696353 \\
\hline L. crassispora & CMW 13488 & Eucalyptus urophylla & Venezuela & DQ103552 & DQ103559 & KU887507 & KU696352 \\
\hline L. euphorbiicola & CMM 3609 & Jatropha curcas & Brazil & KF234543 & KF226689 & KF254926 & N/A \\
\hline L. euphorbiicola & CMW 33350 & Adansonia digitata & Botswana & KU887149 & KU887026 & KU887455 & KU696346 \\
\hline L. euphorbiicola & CMW 36231 & Adansonia digitata & Zimbabwe & KU887187 & KU887063 & KU887494 & KU696347 \\
\hline L. exigua & CBS 137785 & Retama raetam & Tunisia & KJ638317 & KJ638336 & KU887509 & KU696355 \\
\hline L. exigua & BL 184 & Retama raetam & Tunisia & KJ638318 & KJ638337 & N/A & N/A \\
\hline L. gilanensis & IRAN 1523C & Unknown & Iran & GU945351 & GU945342 & KU887511 & KU696357 \\
\hline L. gilanensis & IRAN 1501C & Unknown & Iran & GU945352 & GU945341 & KU887510 & KU696356 \\
\hline
\end{tabular}


L. gonubiensis

L. gonubiensis

L. gravistriata

L. gravistriata

L. hormozganensis

L. hormozganensis

L. hyalina

L. hyalina

L. iraniensis

L. iraniensis

L. iraniensis

L. iraniensis

L. iraniensis

L. laeliocattleyae

L. laeliocattleyae

L. lignicola

L. lignicola

L. macrospora

L. mahajangana

L. mahajangana

L. margaritacea

L. margaritacea

L. mediterranea

L. mediterranea

L. missouriana

L. missouriana

L. parva

L. parva

L. plurivora

L. plurivora

CMW 14077
CMW 14078
CMM 4564
CMM 4565
IRAN 1500C
IRAN 1498C
CGMCC 3.17975
CGMCC 3.18383
IRAN 1520C
IRAN 1502C
CMM 3610
CMW 36237
CMW 36239
CBS 130992
BOT 29
CBS 134112
MFLUCC 11-0656
CMM 3833
CMW 27801
CMW 27818
CBS 122519
CBS 122065
CBS 137783
CBS 137784
UCD 2193MO
UCD 2199MO
CBS 456.78
CBS 494.78
STE-U 5803
STE-U 4583

Syzygium cordatum

Syzygium cordatum

Anacardium humile

Anacardium humile

Olea sp.

Mangifera indica

Acacia confusa

unknown tree

Salvadora persica

Juglans sp.

Jatropha curcas

Adansonia digitata

Adansonia digitata

Mangifera indica

Mangifera indica

dead wood

dead wood

Jatropha curcas

Terminalia catappa

Terminalia catappa

Adansonia gibbosa

Adansonia gibbosa

Quercus ilex

Vitis vinifera

Vitis sp.

Vitis sp.

Cassava field-soil

Cassava field-soil

Prunus salicina

Vitis vinifera

South Africa
South Africa
Brazil
Brazil
Iran
Iran
China
China
Iran
Iran
Brazil
Mozambique
Mozambique
Egypt
Egypt
Thailand
Thailand
Brazil
Madagascar
Madagascar
Western Australia
Western Australia
Italy
Italy
USA
USA
Colombia
Colombia
South Africa
South Africa

AY639595

AY639594

DQ103567

KT250949

KT250947

GU945355

KT250950

KT266812

GU945343

GU945356

GU945344

KX499879

KY767661

GU945348

KX499917

KY751302

GU945347

GU945336

GU945335

KF234544

KF226690

KU887121 KU886998

KU887123 KU887000

JN814397

JN814424

JN814401

JX646797

JN814428

KU887003

JX646798

KF234557

FJ900595

FJ900596

EU144050

EU144051

KJ638312

KJ638311

HQ288225

HQ288226

EF622083

EF622084

EF445362

AY343482
N/A

KF226718

FJ900641

FJ900642

EU144065

EU144066

KJ638331

KJ638330

HQ288267

HQ288268

EF622063

EF622064

EF445395

EF445396
DQ458860

EU673126

N/A

N/A

KU887515

KU887514

KX499992

KY751299

KU887516

KU887517

KF254927

KU887499

KU887501

KU887508

N/A

JX646845

JX646846

KF254941

FJ900630

FJ900631

KU887520

N/A

KU887521

KU887522

HQ288304

HQ288305

KU887523

EU673114

KU887524

KU887525
KU696359

KU696358

N/A

N/A

KU696361

KU696360

KX499955

KY751296

KU696363

KU696362

N/A

KU696348

KU696349

KU696354

N/A

KU696364

N/A

N/A

KU696365

KU696366

KU696367

N/A

KU696368

KU696369

KU696370

KU696371

KU696372

KU696373

KU696374

KU696375 


\begin{tabular}{|c|c|c|c|c|c|c|c|}
\hline L. pontae & CMM 1277 & Spondias purpurea & Brazil & KT151794 & KT151791 & KT151797 & N/A \\
\hline L. pseudotheobromae & CBS 116459 & Gmelina arborea & Costa Rica & EF622077 & EF622057 & EU673111 & KU696376 \\
\hline L. pseudotheobromae & CGMCC 3.18047 & Pteridium aquilinum & China & KX499876 & KX499914 & KX499989 & KX499952 \\
\hline L. pyriformis & CBS 121770 & Acacia mellifera & Namibia & EU101307 & EU101352 & KU887527 & KU696378 \\
\hline L. pyriformis & CBS 121771 & Acacia mellifera & Namibia & EU101308 & EU101353 & KU887528 & KU696379 \\
\hline L. rubropurpurea & WAC 12535 & Eucalyptus grandis & Australia & DQ103553 & DQ103571 & EU673136 & KU696380 \\
\hline L. rubropurpurea & WAC 12536 & Eucalyptus grandis & Australia & DQ103554 & DQ103572 & KU887530 & KU696381 \\
\hline L. sterculiae & CBS 342.78 & Sterculia oblonga & Germany & KX464140 & KX464634 & KX464908 & KX463989 \\
\hline L. subglobosa & CMM 3872 & Jatropha curcas & Brazil & KF234558 & KF226721 & KF254942 & N/A \\
\hline L. subglobosa & CMM 4046 & Jatropha curcas & Brazil & KF234560 & KF226723 & KF254944 & N/A \\
\hline L. thailandica & CPC 22795 & Mangifera indica & Thailand & KJ193637 & KJ193681 & N/A & N/A \\
\hline L. thailandica & CPC 22755 & Phyllanthus acidus & Thailand & KM006433 & KM006464 & N/A & N/A \\
\hline L. thailandica & CGMCC 3.18382 & Podocarpus macrophyllus & China & KY767662 & KY751303 & KY751300 & KY751297 \\
\hline L. thailandica & CGMCC 3.18384 & Albizia chinensis & China & KY767663 & KY751304 & KY751301 & KY751298 \\
\hline L. theobromae & CBS 164.96 & Fruit along coral reef coast & Papua New Guinea & AY640255 & AY640258 & KU887532 & KU696383 \\
\hline L. theobromae & CBS 111530 & Unknown & Unknown & EF622074 & EF622054 & KU887531 & KU696382 \\
\hline L. venezuelensis & WAC 12539 & Acacia mangium & Venezuela & DQ103547 & DQ103568 & KU887533 & KU696384 \\
\hline L. venezuelensis & WAC 12540 & Acacia mangium & Venezuela & DQ103548 & DQ103569 & KU887534 & N/A \\
\hline L. viticola & UCD 2553AR & Vitis sp. & USA & HQ288227 & HQ288269 & HQ288306 & KU696385 \\
\hline L. viticola & UCD 2604MO & Vitis sp. & USA & HQ288228 & HQ288270 & HQ288307 & KU696386 \\
\hline L. vitis & CBS 124060 & Vitis vinifera & Italy & KX464148 & KX464642 & KX464917 & KX463994 \\
\hline
\end{tabular}

Table 2 A morphological comparison of Lasiodiplodia spp.

\begin{tabular}{|c|c|c|c|c|c|c|c|c|c|c|c|}
\hline \multirow[t]{2}{*}{ Species } & \multicolumn{3}{|c|}{ Paraphyses } & \multicolumn{2}{|c|}{ Conidiogenous cells } & \multicolumn{5}{|c|}{ Conidia } & \multirow[t]{2}{*}{ References } \\
\hline & $\mathbf{B}^{1}$ & $\mathrm{Se}^{2}$ & Size $(\mu \mathrm{m})$ & $\mathbf{A n}^{3}$ & Size $(\mu \mathrm{m})$ & $\mathrm{Se}$ & Size $(\mu \mathrm{m})$ & $\mathbf{L}^{4} / \mathbf{W}^{4}$ & $\mathrm{Cl}^{5}$ & PT $^{6}$ & \\
\hline L. avicenniae & $\mathrm{UN}^{1}$ & $\mathrm{~S}^{2}$ & $\leqslant 170,2-4$ & $1-2$ & $6-15 \times 3-6$ & 1 & $19-30 \times 9-15$ & $\mathrm{UN}$ & $\mathrm{Br}^{5}$ & $\mathrm{UN}$ & Osorio et al. (2017) \\
\hline L. brasiliense & UN & $A^{2}$ & UN & UN & UN & 1 & $22.7-29.2 \times 11.7-17.0$ & UN & UN & UN & Netto et al. (2014) \\
\hline L. bruguierae & $\mathrm{NO}^{1}$ & NO & NO & UN & $11-23 \times 2.7-5$ & 1 & $19-32 \times 11-15$ & UN & $\mathrm{DB}^{5}$ & UN & Osorio et al. (2017) \\
\hline L. caatinguensis & $\mathrm{B}$ & UN & $31-60 \times 2-5$ & UN & $7-15 \times 2-6$ & 1 & $13-20.2 \times 10.1-12.5$ & 1.54 & DB & UN & Coutinho et al. (2017) \\
\hline
\end{tabular}




\begin{tabular}{|c|c|c|c|c|c|c|c|c|c|c|c|}
\hline L. chinensis & $\mathrm{NB}^{1}$ & $1-9$ & $\leqslant 99,3-7$ & NO & $10-15 \times 4-6$ & 1 & $19-25 \times 12-14$ & 1.75 & UN & UN & Dou et al. (2017) \\
\hline L. citricola & $\mathrm{OB}^{1}$ & $1-5$ & $\leqslant 125,3-4$ & $1-2$ & $11-16 \times 3-5$ & 1 & $20-31 \times 11-19$ & 1.6 & $\mathrm{UN}$ & UN & Abdollahzadeh et al. (2010) \\
\hline L. crassispora & UN & $S$ & $21-66 \times 2-4$ & UN & $6-19 \times 3-7$ & 1 & $27-33 \times 14-17$ & 1.8 & UN & UN & Burgess et al. (2006) \\
\hline L. euphorbiicola & OB & $S$ & $\leqslant 76,2-4$ & UN & $5-15 \times 3-4$ & 1 & $15-23 \times 9-12$ & UN & DB & UN & Machado et al. (2014) \\
\hline (L. marypalme) & UN & $\mathrm{A}$ & $\mathrm{UN}$ & UN & UN & 1 & $19.1-28.5 \times 10-15.3$ & UN & UN & UN & Netto et al. (2014) \\
\hline L. exigua & UN & $\mathrm{MS}^{2}$ & $61-99 \times 2-3$ & UN & $12-19 \times 3-5$ & 1 & $19.6-24.3 \times 10.8-13.3$ & 1.8 & DB & UN & Linaldeddu et al. (2015) \\
\hline (L. americana) & OB & $1-3$ & $\leqslant 90,2-3.5$ & $1-2$ & $10-18 \times 3-5$ & 1 & $14.0-24.5 \times 10.5-15.0$ & 1.57 & DB & UN & Chen et al. (2015) \\
\hline L. gilanensis & OB & $1-3$ & $\leqslant 95,2-4$ & UN & $11-18 \times 3-5$ & 1 & $25-39 \times 14.5-19$ & 1.9 & UN & UN & Abdollahzadeh et al. (2010) \\
\hline L. gonubiensis & UN & A & $14-65 \times 1.5-3$ & $\mathrm{UN}$ & $6.5-18 \times 1-4.5$ & $1-3$ & $28-39 \times 14-21$ & 1.9 & $\mathrm{Ci}^{5}-\mathrm{Se}^{5}$ & UN & Pavlic et al. (2004) \\
\hline L. gravistriata & UN & A & UN & UN & $9-14 \times 3-5$ & 1 & $24.5-28.5 \times 10.5-16$ & UN & UN & UN & Netto et al. (2017) \\
\hline L. hormozganensis & OB & $1-7$ & $\leqslant 83,2-4$ & UN & $9-15 \times 3-5$ & 1 & $15.3-25.2 \times 11-14$ & 1.7 & UN & UN & Abdollahzadeh et al. (2010) \\
\hline L. hyalina & OB & $1-7$ & $24-82 \times 3-7$ & NO & $8-20 \times 4-7$ & 1 & $19-28 \times 12-16$ & 1.77 & $\mathrm{~PB}^{5}$ & $3 \mathrm{MO}^{6}$ & Present study \\
\hline L. indica & OB & $S$ & $\leqslant 120,1.5-3.5$ & UN & $\begin{array}{l}8.5-17.5 \times 1.5- \\
4\end{array}$ & $1-2$ & $20-38 \times 11-20.5$ & UN & DB & UN & Prasher \& Singh (2014) \\
\hline L. iraniensis & OB & $1-6$ & $\leqslant 127,2-4$ & UN & $9-16 \times 3-5$ & 1 & $15.3-29.7 \times 11-14$ & 1.6 & UN & UN & Abdollahzadeh et al. (2010) \\
\hline (L. jatrophicola) & OB & S & $\leqslant 70,3$ & UN & $7-15 \times 2-5$ & 1 & $22-26 \times 14-17$ & UN & DB & UN & Machado et al. (2014) \\
\hline L. laeliocattleyae & UN & A & $\leqslant 95,2-3$ & $\mathrm{UN}$ & $11-14 \times 3-4$ & 1 & $18-27.4 \times 11.7-17.2$ & 1.6 & DB & UN & Rodríguez-Gálvez et al. (2017) \\
\hline (L. egyptiacae) & UN & $\mathrm{A}$ & $\leqslant 57,2-3$ & $1-2$ & $5-11 \times 3-5$ & 1 & $17-27 \times 11-13$ & 2 & $\mathrm{Br}$ & UN & Ismail et al. (2012) \\
\hline L. lignicola & UN & A & $\leqslant 15$ & UN & $\begin{array}{l}10-15 \times 2.5- \\
3.5\end{array}$ & UN & $15-17.5 \times 8-11$ & 1.7 & DB & UN & Phillips et al. (2013) \\
\hline L. macrospora & NB & $S$ & $\leqslant 105,3-4$ & UN & $8-20 \times 2.5-4$ & $\begin{array}{l}1-3 \\
\text { young, } 1 \\
\text { mature }\end{array}$ & $28-35 \times 15-17$ & UN & DB & UN & Machado et al. (2014) \\
\hline L. mahajangana & UN & A & $27.5-66 \times 2-5$ & UN & $10-26 \times 3-6$ & 1 & $13.5-21.5 \times 10-14$ & 1.4 & UN & UN & Begoude et al. (2010) \\
\hline L. margaritacea & UN & $1-2$ & $19-54 \times 1.5-3$ & UN & $6-19.5 \times 2-4.5$ & 1 & $12-19 \times 10-12.5$ & 1.3 & $\mathrm{Ci}-\mathrm{Se}$ & UN & Pavlic et al. (2008) \\
\hline L. mediterranea & OB & $\mathrm{S}$ & $66-107 \times 2-3$ & UN & $11-16 \times 3-5$ & $1-2$ & $26.3-37 \times 13.5-18$ & 1.9 & DB & $\mathrm{LT}^{6}$ & Linaldeddu et al. (2015) \\
\hline L. missouriana & NB & $\mathrm{A}$ & $\leqslant 55,2-3$ & UN & UN & 1 & $16.1-21 \times 8.1-11.8$ & 1.89 & DB & UN & Urbez-Torres et al. (2012) \\
\hline L. parva & UN & $\mathrm{S}$ & $\leqslant 105,3-4$ & $1-2$ & UN & 1 & $15.5-24.5 \times 10-14.5$ & 1.8 & dark & LT & Alves et al. (2008) \\
\hline L. plurivora & OB & $1-6$ & $\leqslant 130,2-10$ & UN & $8-13 \times 4-7$ & 1 & $22-35 \times 13-18.5$ & 1.9 & $\mathrm{Br}$ & UN & Damm et al. (2007) \\
\hline L. pontae & B & UN & $19-46 \times 2-3$ & UN & $6-16 \times 3-5$ & 1 & $16-26 \times 9.6-15$ & 1.74 & $\mathrm{Br}$ & UN & Coutinho et al. (2017) \\
\hline$L$ & OB & $\mathrm{RS}^{2}$ & $\leqslant 58,3-4$ & $1-2$ & UN & 1 & $22.5-33 \times 13.5-20$ & 1.7 & DB & $\mathrm{LT}$ & Alves et al. (2008) \\
\hline
\end{tabular}


pseudotheobromae

\begin{tabular}{|c|c|c|c|c|c|c|c|c|c|c|c|}
\hline L. pyriformis & UN & A & $\begin{array}{l}27-33.5 \times 1.5- \\
2\end{array}$ & UN & $7-16 \times 2.5-6.5$ & A & $19-28 \times 13.5-21.5$ & 1.3 & $\mathrm{Se}$ & $4 \mathrm{wk}^{6}$ & Slippers et al. (2014) \\
\hline L. rubropurpurea & UN & A & $\begin{array}{l}30-58 \times 1.5- \\
3.5\end{array}$ & 1 & $7-15 \times 3-5$ & 1 & $24-33 \times 13-17$ & 1.9 & UN & UN & Burgess et al. (2006) \\
\hline L. sterculiae & NO & NO & NO & $1-2$ & $\begin{array}{l}7-12 \times 2.5- \\
3.5\end{array}$ & UN & $14-16 \times 10-11$ & UN & $\mathrm{H}^{5}$ & UN & Yang et al. (2017) \\
\hline L. subglobosa & NB & A & $\leqslant 41,2-3$ & UN & $8-18 \times 3-4.5$ & 1 & $16-23 \times 11-17$ & UN & DB & UN & Machado et al. (2014) \\
\hline L. thailandica & UN & $1-3$ & $25-51 \times 1-1.5$ & UN & $8-9 \times 2-4$ & 1 & $20-26 \times 12-16$ & UN & $\mathrm{PB}$ & UN & Trakunyingcharoen et al.(2015) \\
\hline L. theobromae & OB & S & $\leqslant 55,3-4$ & $1-2$ & UN & 1 & $19-32.5 \times 12-18.5$ & 1.9 & DB & $\mathrm{LT}$ & Alves et al. (2008) \\
\hline L. venezuelensis & UN & $S$ & $12-45 \times 1.5-5$ & $\mathrm{UN}$ & $5-15 \times 3-5$ & 1 & $26-33 \times 12-15$ & 2.1 & UN & UN & Burgess et al. (2006) \\
\hline L. viticola & NB & A & $\leqslant 60,2-3$ & UN & UN & 1 & $17-23 \times 8-11$ & 2.05 & DB & UN & Urbez-Torres et al. (2012) \\
\hline L. vitis & NB & A & $\leqslant 60,2-3$ & $1-3$ & $5-15 \times 5-8$ & 1 & $26-28 \times 15-16$ & UN & DB & UN & Yang et al. (2017) \\
\hline L. sp. & OB & $\mathrm{RS}$ & $\leqslant 61,2-3$ & UN & $11-15 \times 3-4$ & 1 & $16-26 \times 9-16$ & 1.7 & $\mathrm{Br}$ & $\mathrm{SA}^{6}$ & Rodríguez-Gálvez et al. (2017) \\
\hline
\end{tabular}

Note: ${ }^{1} \mathrm{~B}=$ branch, $\mathrm{UN}=$ unknown, $\mathrm{NO}=$ not observed, $\mathrm{OB}=$ occasionally branched=rarely branched=sometimes branched, $\mathrm{NB}=$ not branched.

${ }^{2} \mathrm{Se}=$ septation, $\mathrm{S}=$ septate, $\mathrm{A}=$ aseptate, $\mathrm{MS}=$ mostly septate, $\mathrm{RS}=$ rarely septate=mostly aseptate.

${ }^{3}$ An=annellations.

${ }^{4} \mathrm{~L}=$ Length, W=Width.

${ }^{5} \mathrm{Cl}=$ colour, $\mathrm{Br}=$ brown, $\mathrm{DB}=$ dark brown, $\mathrm{Ci}=$ cinnamon, $\mathrm{Se}=$ sepia, $\mathrm{PB}=$ pale brown, $\mathrm{H}=$ hyaline.

${ }^{6} \mathrm{PT}=$ schedule of getting pigmentation, $\mathrm{MO}=$ month, $\mathrm{LT}=\mathrm{a}$ long time, $\mathrm{wk}=$ week, $\mathrm{SA}=$ soon after being formed. 


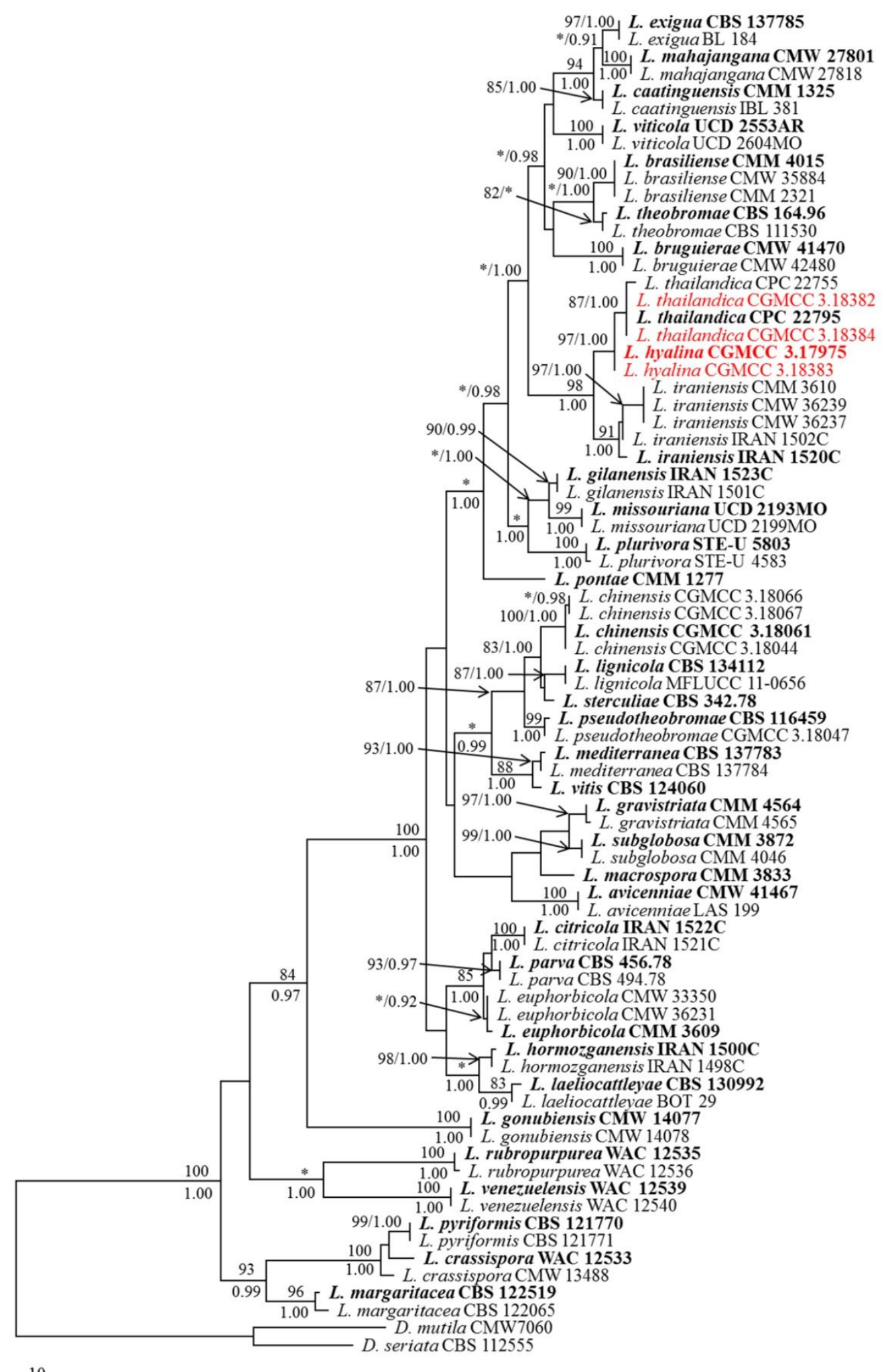

Figure 1 - Maximum parsimony tree generated from sequence analysis of the combined ITS nrDNA, tefl- $\alpha, T U B$ and RPB2 dataset. Designated out group taxon is Diplodia mutila and D. seriata. Bootstrap support values for maximum parsimony (MP) greater than $80 \%$ are shown above at the nodes. Bayesian bootstrap (BP) posterior probability scores above 0.90 are shown under the branches (* $=$ MP value less than $80 \%$ or BP value less than 0.90$)$. The species characterized in this study are in red, and the ex-type strains are in boldface. 

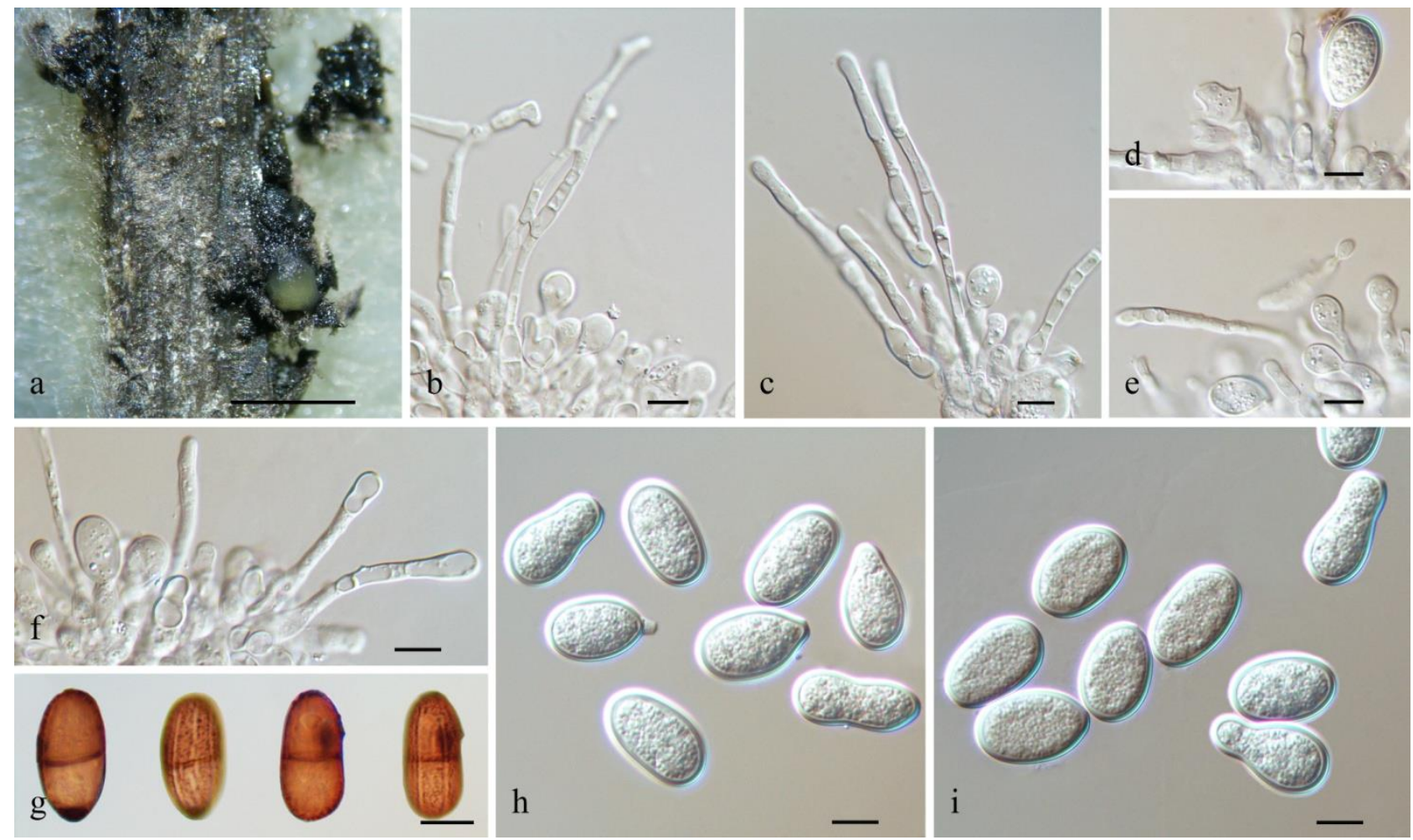

Figure 2 - Lasiodiplodia hyalina (From holotype, CGMCC 3.17975) a. Conidiomata formed on pine needles in culture. $b$. The ladder shaped paraphyses. $c-f$. Conidia developing on conidiogenous cells between paraphyses. g. Mature, 1-septate conidia with longitudinal striations. h, i. Hyaline conidia. Scale bars: $\mathrm{a}=1 \mathrm{~mm} ; \mathrm{b}-\mathrm{i}=10 \mu \mathrm{m}$.

\section{Acknowledgements}

This study was financially supported by NSFC Projects of National Natural Science Foundation of China (General Program, 31370063), International Cooperation and Exchanges (31461143028) and National Science and Technology Foundation Project (2014FY210400).

\section{References}

Abdollahzadeh J, Javadi A, Goltapeh EM, Zare R et al. 2010 - Phylogeny and morphology of four new species of Lasiodiplodia from Iran. Persoonia 25, 1-10.

Alves A, Crous PW, Correia A, Phillips AJL. 2008 - Morphological and molecular data reveal cryptic species in Lasiodiplodia theobromae. Fungal Diversity 28, 1-13.

Begoude BAD, Slippers B, Wingfield MJ, Roux J. 2010 - Botryosphaeriaceae associated with Terminalia catappa in Cameroon, South Africa and Madagascar. Mycological Progress 9, 101-123.

Burgess TI, Barber PA, Mohali S, Pegg G et al. 2006 - Three new Lasiodiplodia spp. from the tropics, recognized based on DNA sequence comparisons and morphology. Mycologia 98, $423-435$.

Chen SF, Li GQ, Liu FF, Michailides TJ. 2015 - Novel species of Botryosphaeriaceae associated with shoot blight of pistachio. Mycologia 107, 780-792.

Clendenin I. 1896 - Lasiodiplodia Ellis \& Everh. n. gen. Botanical Gazette Crawfordsville 21, 92 93.

Coutinho IBL, Freire FCO, Lima CS, Lima JS et al. 2017 - Diversity of genus Lasiodiplodia, associated with perennial tropical fruit plants in northeastern Brazil. Plant Pathology 66, 90104. 
Cruywagen EM, Slippers B, Roux J, Wingfield MJ. 2017 - Phylogenetic Species Recognition and hybridisation in Lasiodiplodia: A case study on species from baobabs. Fungal Biology 121, 420-436.

Dai YC, Cui BK, Liu XY. 2010 - Bondarzewia podocarpi, a new and remarkable polypore from tropical China. Mycologia 102, 881-886.

Dai YC, Cui BK, Yuan HS, 2009 - Trichaptum (Basidiomycota, Hymenochaetales) from China with a description of three new species. Mycological Progress 8, 281-287.

Dai YC, Cui BK, Yuan HS, He SH et al. 2011 - Wood-inhabiting fungi in southern China 4. Polypores from Hainan Province. Annales Botanici Fennici 48, 219-231.

Damm U, Crous PW, Fourie PH. 2007 - Botryosphaeriaceae as potential pathogens of Prunus species in South Africa, with descriptions of Diplodia africana and Lasiodiplodia plurivora sp. nov. Mycologia 99, 664-680.

Denman S, Crous PW, Taylor JE, Kang JC et al. 2000 - An overview of the taxonomic history of Botryosphaeria, and a re-evaluation of its anamorphs based on morphology and ITS rDNA phylogeny. Studies in Mycology 45, 129-140.

Dou ZP, He W, Zhang Y. 2017 - Lasiodiplodia chinensis sp. nov., a new holomorphic species from China. Mycosphere 8(2), 521-532.

Glass NL, Donaldson GC. 1995 - Development of primer sets designed for use with the PCR to amplify conserved genes from filamentous Ascomycetes. Applied \& Environmental Microbiology 61, 1323-1330.

Huelsenbeck JP, Ronquist F. 2001 - MRBAYES: Bayesian inference of phylogenetic trees. Bioinformatics 17, 754-755.

Huelsenbeck JP, Ronquist F. 2005 - Bayesian analysis of molecular evolution using MrBayes. In: Statistical methods in molecular evolution (ed Nielsen R.), Springer, New York: 183-232.

Ismail AM, Cirvilleri G, Polizzi G, Crous PW et al. 2012 - Lasiodiplodia species associated with dieback disease of mango (Mangifera indica) in Egypt. Australasian Plant Pathology 41, 649-660.

Li GQ, Arnold RJ, Liu FF, Li JQ et al. 2015 - Identification and Pathogenicity of Lasiodiplodia Species from Eucalyptus urophylla $\times$ grandis, Polyscias balfouriana and Bougainvillea spectabilis in Southern China. Journal of Phytopathology 163, 956-967.

Linaldeddu BT, Deidda A, Scanu B, Franceschini A et al. 2015 - Diversity of Botryosphaeriaceae species associated with grapevine and other woody hosts in Italy, Algeria and Tunisia, with descriptions of Lasiodiplodia exigua and Lasiodiplodia mediterranea sp. nov. Fungal Diversity 71, 201-214.

Luo M, Dong ZY, Bin SY, Lin JT. 2011 - First report of fruit rot disease on pomelo caused by Lasiodiplodia theobromae in China. Plant Disease 95, 1190-1190.

Machado AR, Pinho DB, Pereira OL. 2014 - Phylogeny, identification and pathogenicity of the Botryosphaeriaceae associated with collar and root rot of the biofuel plant Jatropha curcas in Brazil, with a description of new species of Lasiodiplodia. Fungal Diversity 67, 1-17.

Netto MSB, Assunção IP, Lima GSA, Marques MW et al. 2014 - Species of Lasiodiplodia associated with papaya stem-end rot in Brazil. Fungal Diversity 67, 127-141.

Netto MSB, Lima WG, Correia KC, Silva CFBD et al. 2017 - Analysis of phylogeny, distribution, and pathogenicity of Botryosphaeriaceae species associated with gummosis of Anacardium in Brazil, with a new species of Lasiodiplodia, Fungal Biology 121, 437-451.

Osorio JA, Crous CJ, Beer ZWD, Wingfield MJ et al. 2017 - Endophytic Botryosphaeriaceae, including five new species, associated with mangrove trees in South Africa. Fungal Biology 121, 361-393.

Pavlic D, Slippers B, Coutinho TA, Gryenhout M et al. 2004 - Lasiodiplodia gonubiensis sp. nov., a new Botryosphaeria anamorph from native Syzygium cordatum in South Africa. Studies in Mycology 50, 313-322. 
Pavlic D, Wingfield MJ, Barber P, Slippers B et al. 2008 - Seven new species of the Botryosphaeriaceae from baobab and other native trees in Western Australia. Mycologia 100(6), 851-866.

Phillips AJL, Alves A, Abdollahzadeh J, Slippers B et al. 2013 - The Botryosphaeriaceae: genera and species known from culture. Studies in Mycology 76, 51-167.

Phillips AJL, Alves A, Pennycook SR, Johnston PR et al. 2008 - Resolving the phylogenetic and taxonomic status of dark-spored teleomorph genera in the Botryosphaeriaceae. Persoonia $21,29-55$.

Posada D, Buckley TR. 2004 - Model selection and model averaging in phylogenetics: advantages of Akaike information criterion and Bayesian approaches over likelihood ratio tests. Systematic Biology 53, 793-808.

Prasher IB, Singh G. 2014 - Lasiodiplodia indica -A new species of coelomycetous mitosporic fungus from India. Kavaka 43, 64-69.

Rodríguez-Gálvez E, Guerrero P, Barradas C, Crous PW et al. 2017 - Phylogeny and pathogenicity of Lasiodiplodia, species associated with dieback of mango in Peru. Fungal Biology 121, 437-451.

Ronquist F, Huelsenbeck JP. 2003 - MrBayes3: Bayesian phylogenetic inference under mixed models. Bioinformatics 19, 1572-1574.

Slippers B, Crous PW, Denman S, Coutinho TA et al. 2004 - Combined multiple gene genealogies and phenotypic characters differentiate several species previously identified as Botryosphaeria dothidea. Mycologia 96, 83-101.

Slippers B, Crous PW, Jami F, Groenewald JZ et al. 2017 -Diversity in the Botryosphaeriales: Looking back, looking forward. Fungal Biology 121, 307-321.

Slippers B, Roux J, Wingfield MJ, Walt FJJVD et al. 2014 - Confronting the constraints of morphological taxonomy in the Botryosphaeriales. Persoonia 33, 155-168.

Sutton BC, 1980. The Coelomycetes: Fungi Imperfecti with Pycnidia, Acervuli and Stromata. Kew, UK: Commonwealth Mycological Institute.

Swofford DL. 2002. PAUP*. Phylogenetic Analysis Using Parsimony (*and Other Methods). Version 4. Sinauer Associates, Sunderland, Massachusetts.

Tamura K, Stecher G, Peterson D, Filipski A et al. 2013 - MEGA6: molecular evolutionary genetics analysis version 6.0. Molecular Biology \& Evolution 30, 2725-2729.

Trakunyingcharoen T, Lombard L, Groenewald JZ, Cheewangkoon R et al. 2015 - Caulicolous Botryosphaeriales from Thailand. Persoonia 34, 87-99.

Urbez-Torres JR, Peduto F, Striegler RK, Urrea-Romero KE et al. 2012 - Characterization of fungal pathogens associated with grapevine trunk diseases in Arkansas and Missouri. Fungal Diversity 52(1), 169-189.

White TJ, Bruns T, Lee S, Taylor J. 1990. Amplification and direct sequencing of fungal ribosomal RNA genes for phylogenetics. In: Innis MA, Gelfand DH, Sninsky JJ, White TJ (eds), PCR Protocols: a guide to methods and applications. Academic Press, New York, pp. 315-322.

Yang T, Groenewald JZ, Cheewangkoon R, Jami F et al. 2017 - Families, genera and species of Botryosphaeriales. Fungal Biology 121, 322-346.

Zhang Y, Jeewon R, Fournier J, Hyde KD. 2008 - Multi-gene phylogeny and morphotaxonomy of Amniculicola lignicola: a novel freshwater fungus from France and its relationships to the Pleosporales. Mycological Research 112, 1186-1194.

Zhang Y, Wang HK, Fournier J, Crous PW et al. 2009 - Towards a phylogenetic clarification of Lophiostoma / Massarina and morphologically similar genera in the Pleosporales. Fungal Diversity 38, 225-251.

Zhao JP, Lu Q, Liang J, Decock C et al. 2010 - Lasiodiplodia pseudotheobromae, a new record of pathogenic fungus from some subtropical and tropical trees in southern China. Cryptogamie Mycologie 31, 431-439.

Zhou LW, Dai YC. 2013 - Taxonomy and phylogeny of wood-inhabiting hydnoid Russulales: two new genera, three new species and two new combinations. Mycologia 105, 636-649. 
Zhou S, Stanosz GR. 2001 - Relationships among Botryosphaeria species and associated anamorphic fungi inferred from the analysis of ITS and 5.8S rDNA sequences. Mycologia 93, 516-527. 